\title{
Probing Light New Mediators on Coherent Elastic Neutrino-Nucleus Scattering
}

\author{
M. Demirci and M. F. Mustamin \\ Department of Physics, Karadeniz Technical University, Trabzon, TR61080, Turkey
}

\begin{abstract}
We study the occurrence of light mediators on coherent elastic neutrino-nucleus scattering in the framework of a simplified model. The model includes light mediators in accordance with all possible interactions such as scalar, pseudoscalar, vectorial, axial-vector, and tensorial, and commonly used in explaining solar neutrino phenomena. We show the event rate spectrum using flux from reactor and accelerator neutrino experiment for $\mathrm{keV}, \mathrm{MeV}$, and $\mathrm{GeV}$ mass scale of the new mediators. We then present the $90 \%$ C.L. constraint on parameter of the considered model with the first COHERENT data.
\end{abstract}

Keywords: CEvNS, neutrino, BSM, light mediator

DOI: 10.31526/ACP.BSM-2021.31

\section{INTRODUCTION}

Studying new physics via neutrino interactions with the nucleus has been widely conducted in recent years. Especially the process of coherent elastic neutrino-nucleus scattering (CEvNS), a tree-level process in the standard model (SM), where neutrinos interact with the nucleus as a whole via neutral current [1]. The scattered nucleus recoiled with small energy which is the only observable quantity from the process. CEvNS provides the largest cross-section prediction among other processes involving neutrino so far, yet hard to be witnessed since the low energy requirement of the coherency criteria. The importance of this process may be used on probing proposed beyond SM (BSM) proposals [2], explaining astrophysical phenomena [3], and also observing dark matter candidate [4].

Since its first introduction a few decades ago, the successful observation was recently done by COHERENT collaboration at the Oak National Laboratory using stopped pion [5]. Neutrinos source of this experiment originated from decay at rest pions $(\mathrm{DAR}-\pi)$ with the energy of a few tens $\mathrm{MeV}$, directed towards CsI[Na] scintillator and produce 5 keV energy threshold for Cs [6]. Nevertheless, the criteria of full coherency still unreachable which lie in the lower nuclear recoil energy, around some keV, and predicted to be achieved by reactor neutrino with sensitive detector [7]. Higher energies just break the elastic process, surpassed by inelastic which termed incoherency recently [8]. To pursue these criteria, some proposed advancements have been planned and they run observation now. TEXONO in their advancement set to reach $0.1 \mathrm{keV}$ nuclear threshold with Germanium target [9], where, in their recent update has successfully reached $0.2 \mathrm{keV}$ [10]. The DarkSide collaboration with $0.6 \mathrm{keV}$ energy threshold and liquid Argon aims to study Weak Interacting Massive Particle (WIMP) dark matter [11]. The nuclear power plant as neutrino source is used by CONUS experiment [12].

The possible occurrence of new scalar dan vector mediators from a simplified model has been frequently conducted recently. These light new particles are considered as the extension of the standard neutrino sector as well as explaining dark matter (DM) candidates since its similarity with the formulation of DM direct-detection. They may come from solar and other astrophysical sources and hope to be witnessed in current and future detectors. They can also be observed from artificial sources such as in neutrino accelerator from $\pi$-stopped process in which various neutrino flavors can be detected. Stringent bound parameters have been found from the advancement of CE $v$ NS related experiments regarding these two new light particles [13, 14, 15].

In general, the simplified model may also induce other types of interactions such as pseudoscalar, axial-vector, and also tensorial. In this work, we focus on including these possibilities in the CE $v$ NS framework. The effect of its occurrence is probed using neutrino fluxes from the detector and neutrino accelerator. We consider flux of the CHOOZ experiment [16] with Ge target for the former while the SNS [5] with CsI target for the latter. We use digitalized flux with the corresponding normalization such as used in Ref.[17]. The event rate spectrum from each possible interaction is presented by setting benchmarks for the coupling value, related to the mediator with $\mathrm{keV}, \mathrm{MeV}$, and $\mathrm{GeV}$ mass scale. Particularly to the COHERENT 2017 data, we also give lower bound constraint for each coupling from the new interactions.

In what follows, we first review differential cross-section formulation of the CEvNS process in the SM. Next, the effect of coherency is shown by presenting the form factor for the considered nucleus. The inclusion of the proposed new mediators along with their possible spectrum is then discussed next. Afterwards, we briefly explain the properties of neutrino flux from neutrino reactor and accelerator experiments. We then present the event rates of each contribution and present the $\chi^{2}$-analysis from COHERENT 1-bin data. Finally, we conclude our work. 


\section{FORMULATION OF CE $v$ NS}

In the SM, the neutral current interactions enable low energy neutrinos with $E_{v} \leq 50 \mathrm{MeV}$ to interact coherently with protons and neutrons in a nucleus, which significantly enhance the cross-section for a large nucleus. Regarding the nucleus as a spin- $1 / 2$ particle, the differential cross-section as a function of nuclear recoil energy $T_{N}$ in the SM can be written as [18]

$$
\frac{d \sigma_{S M}}{d T_{N}}=\frac{G_{F}^{2} Q_{S M}^{2} M}{4 \pi}\left(1-\frac{T_{N}}{T_{N_{\max }}}\right)\left|F\left(q^{2}\right)\right|^{2} .
$$

Here, $G_{F}$ denotes the Fermi coupling-constant and $M$ the nucleus mass. $Q_{S M}$ represents the SM weak charge and defined as

$$
Q_{S M}=\mathcal{N}-\left(1-4 \sin ^{2} \theta_{W}\right) \mathcal{Z}
$$

where $\mathcal{N}$ and $\mathcal{Z}$ give respectively the number of neutron and proton of the involved nucleus, and $\sin _{\theta_{W}}$ is the weak angle of the electroweak theory. Updated value of this angle [19] estimated $1-4 \sin ^{2} \theta_{W} \equiv 0.045$, indicating that the process depends quadratically on $\mathcal{N}$ of the target nuclei. This charge characterizes the SM case which comes from vector interaction. The spin- $1 / 2$ approximation only differ on the last term in the kinematics of the cross-section of Eq. 1 with the spin- 0 case, which for small nuclear recoil energy can be neglected. The maximum value of the nucleus recoil energy $T_{N}$, for $E_{v}<<M$, is given by $T_{N m a x}=2 E_{v}^{2} / M$. $E_{v}$ represents the initial neutrino energy. Also, notice that this form is applicable to both neutrino and antineutrino cases. It stems from the parity-conserved nature as neutral $\mathrm{Z}$ boson exchange considered. For new physics beyond the SM, Eq. 1 can be modified but $T_{N \max }$ still holds because it is determined purely from relativistic kinematics. There is usually a detection threshold on $T_{N}$ for all types of detectors, denoted by $T_{t h}$. Therefore, the recoil energy $T_{N}$ of detected events should be in the range $T_{t h} \leq T_{N} \leq T_{N m a x}$ for a given $E_{v}$.

The $F\left(q^{2}\right)$ denotes the nucleus form factor as a function of momentum transfer $q$, which is related by nucleus recoil energy as $-q^{2} \equiv Q^{2}=2 M T_{N}$, and represents the structure of the nucleus. It contains large uncertainty of the nucleon structure. Assumption of the same neutron and proton form factor is also considered. For this work, the Helm parameterization [20] is being implemented as the form factor:

$$
F\left(q^{2}\right)=\frac{3 J_{1}(q R)}{q R} e^{-\frac{1}{2}(q s)^{2}}
$$

Here $J_{1}$ is the spherical Bessel function of the first order, with $R=\sqrt{c^{2}+7 \pi^{2} / 3-5 s^{2}}$ is the radius of a nucleus where $c=$ $\left(1.23 \mathcal{A}^{1 / 3}-6\right) \mathrm{fm}$ is the rms radius with $\mathcal{A}=\mathcal{Z}+\mathcal{N}$, and $s=0.9 \mathrm{fm}$ its thickness. We present in Fig 1 the effect of this form factor for the case of Ge and CsI target, the preferred nucleus in detector (TEXONO, CONUS) and accelerator (COHERENT) neutrino experiment, respectively. Heavier target, CsI, penetrate deeper than the lighter one, Ge. The case of the point nucleus is given by the solid black line.

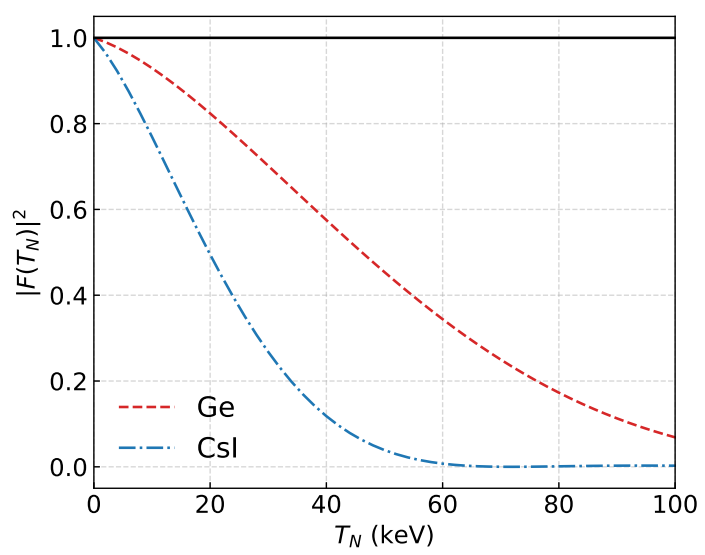

FIGURE 1: Form factor as a function of nuclear threshold.

Since the process occurs at small $T_{N}$, full coherency criteria can be approximated as $F\left(T_{N}\right) \approx 1$. This process indicates that the internal structure of the system, both neutrino and nucleus, is not affected after scattering. This criterion is reached in the small scale of nuclear recoil energy. For example in COHERENT experiment $\left(E_{v}=50 \mathrm{MeV}\right)$ the observed threshold of CsI target is about $20.0 \mathrm{keV}$, while for $\mathrm{CHOOZ}\left(E_{v}=10 \mathrm{MeV}\right) 2.9 \mathrm{keV}$. Even at this energy, the pure elastic process may be suppressed and breaks the coherent criteria. Higher energies still counted as neutrino-nucleus interaction, but the inelastic process takes place [8]. From this result, we can see that a low nuclear threshold experiment is needed to fully probe the CE $v$ NS process. 


\section{LIGHT MEDIATORS WITH SIMPLIFIED MODEL}

We use a simplified model that contains scalar, pseudoscalar, vectorial, axial-vector, and tensorial type. The following are the extended SM Lagrangians to the SM, corresponds to each considered interaction:

$$
\begin{gathered}
\mathcal{L}_{S} \supset\left(g_{v s} \bar{v}_{R} v_{L} \mathcal{S}+\text { h.c. }\right)+g_{q s} \bar{q} q \mathcal{S}, \\
\mathcal{L}_{P} \supset\left(g_{v P} \bar{v}_{R} v_{L} \mathcal{P}+\text { h.c. }\right)-i g_{q P} \bar{q} \gamma^{5} q \mathcal{P}, \\
\mathcal{L}_{V} \supset g_{v V} \bar{v}_{L} \gamma^{\mu} v_{L} \mathcal{V}_{\mu}+g_{q V} \bar{q} \gamma^{\mu} q \mathcal{V}_{\mu}, \\
\mathcal{L}_{A} \supset g_{v A} \bar{v}_{L} \gamma^{\mu} v_{L} \mathcal{A}_{\mu}-g_{q A} \bar{q} \gamma^{\mu} \gamma^{5} q \mathcal{A}_{\mu}, \\
\mathcal{L}_{T} \supset g_{v T} \bar{v}_{R} \sigma^{\mu v} v_{L} \mathcal{T}_{\mu v}-g_{q T} \bar{q} \sigma^{\mu v} q \mathcal{T}_{\mu v},
\end{gathered}
$$

where $\sigma_{\mu \nu}=\frac{i}{2}\left[\gamma_{\mu}, \gamma_{\nu}\right]=\frac{i}{2}\left(\gamma_{\mu} \gamma_{\nu}-\gamma_{\nu} \gamma_{\mu}\right)$. The corresponding differential cross-section from these models are

$$
\begin{gathered}
{\left[\frac{d \sigma}{d T_{N}}\right]_{S}=\frac{G_{F}^{2} m_{N}}{4 \pi} \frac{Q_{S}^{2} m_{N} T_{N}}{E_{v}^{2}\left(2 m_{N} T_{N}+m_{S}^{2}\right)^{2}}\left|F\left(q^{2}\right)\right|^{2},} \\
{\left[\frac{d \sigma}{d T_{N}}\right]_{P}=\frac{G_{F}^{2} m_{N}}{4 \pi} \frac{Q_{P}^{2} T_{N}^{2}}{2 E_{v}^{2}\left(2 m_{N} T_{N}+m_{P}^{2}\right)^{2}}\left|F\left(q^{2}\right)\right|^{2},} \\
{\left[\frac{d \sigma}{d T_{N}}\right]_{S M+V}=\frac{G_{F}^{2} m_{N}}{4 \pi}\left(Q_{S M}+\frac{\sqrt{2} Q_{V}}{G_{F}\left(2 m_{N} T_{N}+m_{V}^{2}\right)}\right)^{2}\left(\frac{2 E_{v}^{2}-m_{N} T_{N}}{2 E_{v}^{2}}\right)\left|F\left(q^{2}\right)\right|^{2},} \\
{\left[\frac{d \sigma}{d T_{N}}\right]_{A}=\frac{G_{F}^{2} m_{N}}{4 \pi} \frac{Q_{A}^{2}\left(2 E_{v}^{2}+m_{N} T_{N}\right)}{E_{v}^{2}\left(2 m_{N} T_{N}+m_{A}^{2}\right)^{2}}\left|F\left(q^{2}\right)\right|^{2}} \\
{\left[\frac{d \sigma}{d T_{N}}\right]_{T}=\frac{G_{F}^{2} m_{N}}{4 \pi} \frac{32 Q_{T}^{2}\left(4 E_{v}^{2}-m_{N} T_{N}\right)}{E_{v}^{2}\left(2 m_{N} T_{N}+m_{T}^{2}\right)^{2}}\left|F\left(q^{2}\right)\right|^{2} .}
\end{gathered}
$$

The charge for each cases has $Q_{i}=g_{v i} g_{N i} / G_{F}$, with $i=S, P, V, A, T$, form. $g_{v i}$ denotes new mediator coupling to neutrino, while $g_{N i}$ to nucleus. The form for each interaction type are obtained from Ref.[21]. For the scalar case:

$$
g_{N S}=\mathcal{Z} \sum_{q=d, u, s} g_{q S} f_{T q}^{p} \frac{m_{p}}{m_{q}}+\mathcal{N} \sum_{q=d, u, s} g_{q S} f_{T q}^{n} \frac{m_{n}}{m_{q}}
$$

for the pseudoscalar case:

$$
g_{N P}=\mathcal{Z} \sum_{q=d, u, s} g_{q P} \frac{m_{p}}{m_{q}}\left(1-\sum_{q} \frac{\bar{m}}{m_{q}}\right) \Delta_{q}^{p}+\mathcal{N} \sum_{q=d, u, s} g_{q N} \frac{m_{n}}{m_{q}}\left(1-\sum_{q} \frac{\bar{m}}{m_{q}}\right) \Delta_{q}^{n},
$$

with $\bar{m}=\left(m_{u}+m_{d}+m_{s}\right) /\left(m_{u} m_{d} m_{s}\right)$; for the vectorial case:

$$
g_{N V}=3 g_{q V}(\mathcal{Z}+\mathcal{N})
$$

for the axial-vector case:

$$
g_{N A}=\mathcal{Z} S_{p} \sum_{q=d, u, s} g_{q A} \Delta_{q}^{p}+\mathcal{N} S_{n} \sum_{d, u, s} g_{q A} \Delta_{q}^{n}
$$

with $S_{p}$ and $S_{n}=1$ related to the nucleon spin as $S_{p, n} \equiv 2 s_{\mu}$ are set to unity; and for the tensorial case:

$$
g_{N T}=\mathcal{Z} \sum_{q=d, u, s} \delta_{q}^{p}+\mathcal{N} \sum_{q=d, u, s} \delta_{q}^{n} .
$$

In the above relations, we set $m_{p}=938.3 \mathrm{MeV}$ and $m_{n}=939.6 \mathrm{MeV}$ for the proton and neutron mass, $m_{u}=2.2 \mathrm{MeV}, m_{d}=4.7$ $\mathrm{MeV}$, and $m_{s}=96 \mathrm{MeV}$ for the light quarks, and for the other parameter is given in Table 1. 


\begin{tabular}{crr}
\hline Parameters & Value & Source \\
\hline \hline$f_{T_{u}}^{p}$ & 0.0208 & {$[22]$} \\
$f_{T_{u}}^{n}$ & 0.0189 & {$[22]$} \\
$f_{T_{d}}^{p}$ & 0.0411 & {$[22]$} \\
$f_{T_{d}}^{n}$ & 0.0451 & {$[22]$} \\
$f_{T_{s}}^{p}=f_{T_{s}}^{n}$ & 0.043 & {$[23]$} \\
\hline$\Delta_{u}^{p}=\Delta_{u}^{n}$ & 0.77 & {$[24]$} \\
$\Delta_{d}^{p}=\Delta_{d}^{n}$ & -0.40 & {$[24]$} \\
$\Delta_{s}^{p}=\Delta_{s}^{n}$ & -0.12 & {$[24]$} \\
\hline$\delta_{u}^{p}=\delta_{d}^{n}$ & 0.84 & {$[25]$} \\
$\delta_{d}^{p}=\delta_{u}^{n}$ & -0.23 & {$[25]$} \\
$\delta_{s}^{p}=\delta_{s}^{n}$ & -0.05 & {$[25]$} \\
\hline
\end{tabular}

TABLE 1: Coupling parameters for the simplified model.
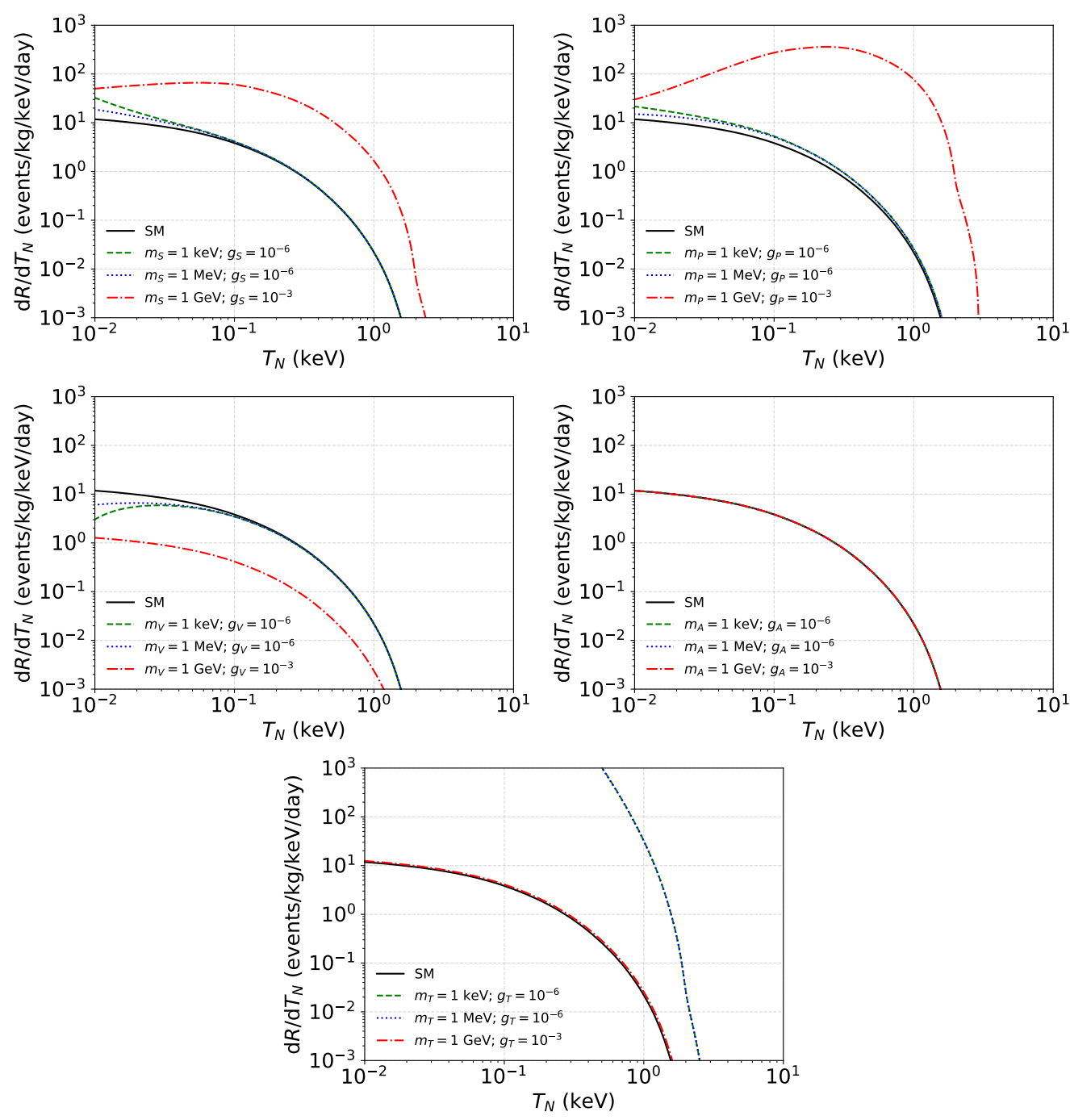

FIGURE 2: The spectrum of differential rate from reactor neutrino expressed for the SM plus the scalar (top-left), pseudoscalar (topright), vectorial (mid-left), axial-vector (mid-right), and tensorial (bottom) new interactions. Three different mass scale are chosen to indicate the new physics effect. 

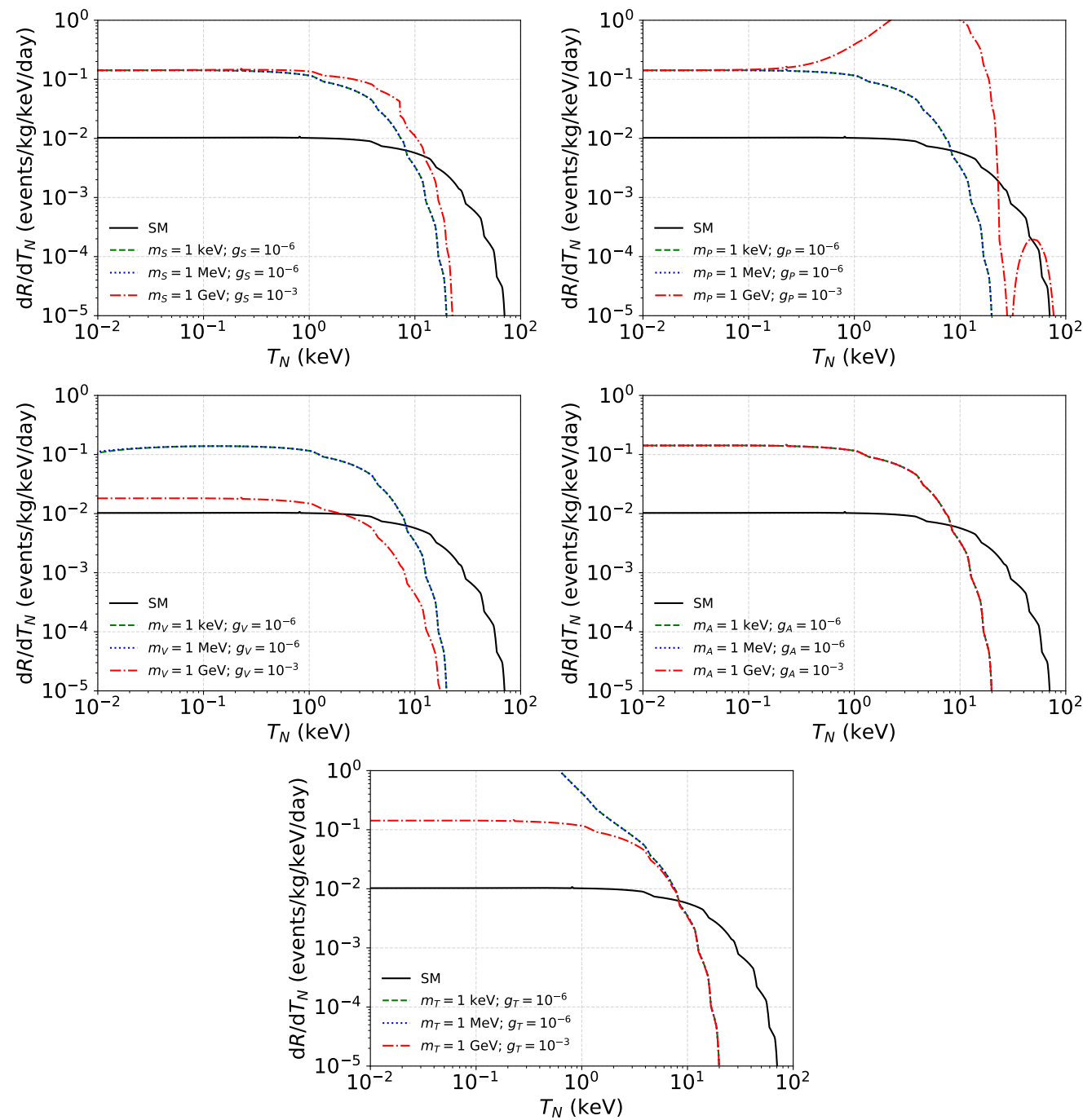

FIGURE 3: The spectrum of differential rate from accelerator neutrino expressed for the SM plus the scalar (top-left), pseudoscalar (top-right), vectorial (mid-left), axial-vector (mid-right), and tensorial (bottom) new interactions. Three different mass scale are chosen to indicate the new physics effect.

\section{NUMERICAL RESULTS}

\subsection{Differential Rate}

Indication of the occurrence of the new interactions is shown as the differential rate of the CEvNS process. The differential rate is obtained using

$$
\left[\frac{d R}{d T_{N}}\right]_{S M+i}=\frac{1}{m_{t a r}} \int_{E_{v_{\min }}}^{E_{v_{\max }}} d E_{v} \Phi \frac{d \sigma_{S M+i}}{d T_{N}}
$$

where the differential cross-section consists of contribution from the new interaction $i$, added with the SM. $\Phi$ denotes neutrino flux. For this work, we consider the neutrino flux from $\mathrm{CHOOZ}$ reactor and SNS accelerator. The effect of the new interactions is shown by setting the new mass scale to be $1 \mathrm{keV}, 1 \mathrm{MeV}$, and $1 \mathrm{GeV}$. Accordingly, we set $10^{-6}$ for the coupling of the keV and MeV scales and $10^{-3}$ for the GeV scale.

The predicted differential rates for the SM and each possible mediators for the reactor case are given in Fig.2. Except for the vectorial case, the new interactions provide a larger spectrum or at least overlap with the SM prediction (solid black line). In the scalar and pseudoscalar, the GeV (dot-dashed red line) mediator gives a larger deviation from the SM spectrum comparing to the $\mathrm{keV}$ (dashed blue) and $\mathrm{MeV}$ (dotted green) ones that only slightly deviate for $T_{N} \sim 0.1 \mathrm{keV}$ or lower. Meanwhile, for the vectorial case, all new interactions tend to be lower than the SM prediction with the GeV gives the lowest spectrum. In the axial-vector case, all new interactions have the same spectrum as the SM one. Finally, for the tensorial, the GeV overlap with the SM and the keV and $\mathrm{MeV}$ diverge for $T_{N}<1 \mathrm{keV}$.

The effect of the new interactions in the accelerator neutrino is shown in Fig.3. As we can see, the spectrum is smaller than from the reactor. In general, the new interaction gives a larger spectrum and decline earlier than the SM. The GeV mediator gives 
the largest spectrum for the case of scalar and pseudoscalar than the $\mathrm{keV}$ and $\mathrm{MeV}$. In the pseudoscalar case, the spectrum shows fluctuation at $T_{N}>1 \mathrm{keV}$ which comes from the form factor. In the vectorial case, the GeV mediator gives a lower effect than the $\mathrm{keV}$ and $\mathrm{MeV}$ mediator. In the axial-vector, all new mediators overlap. For the tensorial case, the GeV gives a stable spectrum at low energy while the $\mathrm{keV}$ and $\mathrm{MeV}$ diverge for $T_{N}<1 \mathrm{keV}$.

\subsection{Coupling vs mass analysis}

From Eq.19, we can obtain the number of events observed by using

$$
N_{x}=m_{\text {tar }} f_{x} t \int_{T_{N_{\min }}}^{T_{N_{\max }}} d T_{N} \frac{d R}{d T_{N}} \epsilon\left(T_{N}\right),
$$

where $m_{t a r}$ is the target mass, $t$ is the run-time of the observation, and $f_{A}$ is the mass fraction of the considered nucleus. For the COHERENT case, we use CsI nucleus with $m_{t a r}=14.6 \mathrm{~kg}$ and $t=308.1$ days. Another important quantity is the number of photo electron per $\mathrm{keV}$, which is taken to be 1.17 from the 2017 COHERENT data.
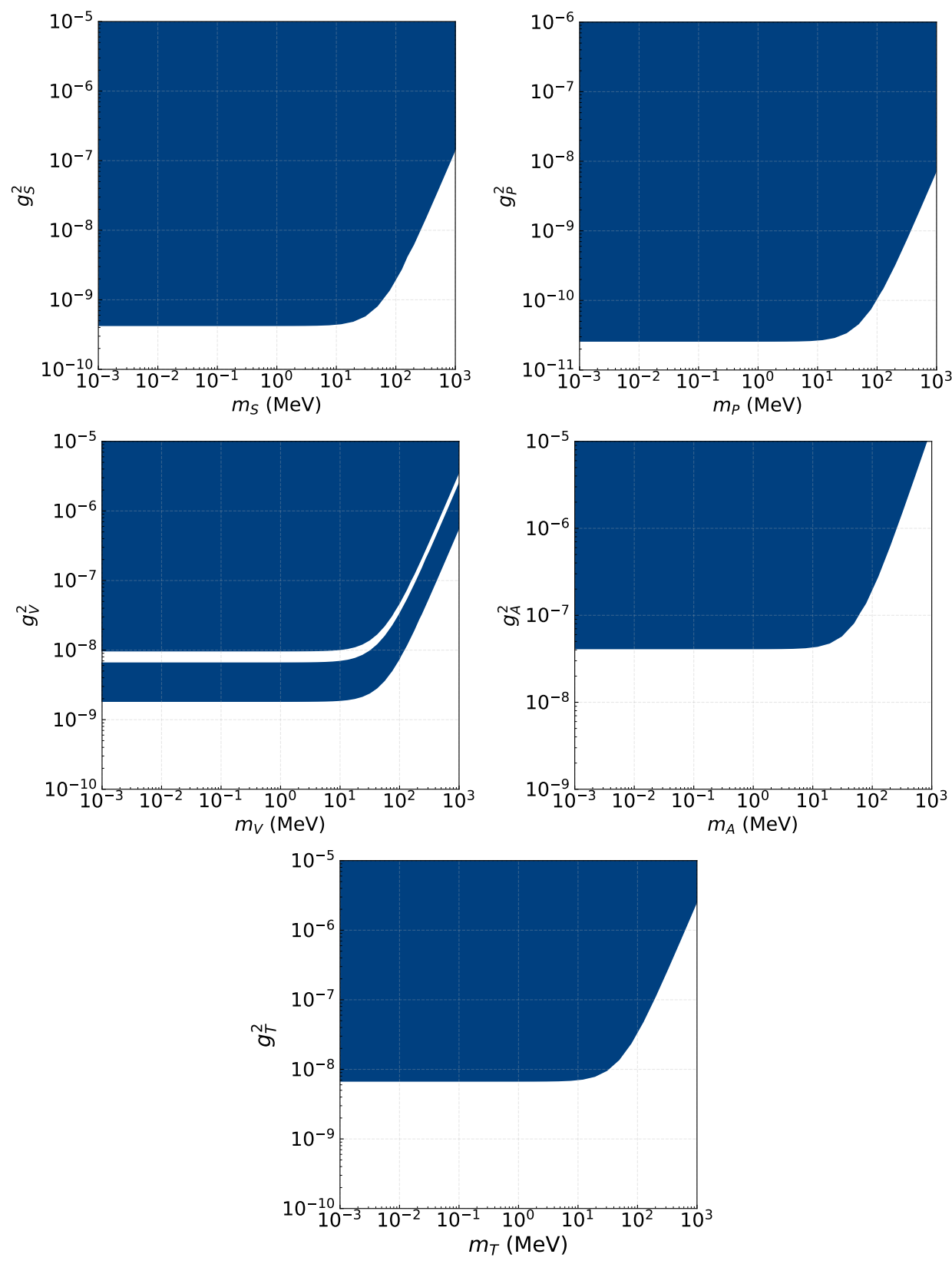

FIGURE 4: 90\% C.L. constraint of scalar (top-left), pseudoscalar (top-right), vectorial (mid-left), axial-vector (mid-right), and tensorial (bottom) coupling vs mass of the proposed new mediators. 
We use the suggested $\chi^{2}$-function from the COHERENT experiment to analyze the lower bound constraint [11]:

$$
\chi^{2}=\frac{\left[N_{o}-N_{\exp }(1+a)-N_{B}(1+b)\right]^{2}}{\left(N_{o}+N_{B}+2 N_{S S}\right)}+\left(\frac{a}{\sigma_{a}}\right)^{2}+\left(\frac{b}{\sigma_{b}}\right)^{2} .
$$

In this equation, $N_{o}$ is the observed CE $v$ NS signal (142), $N_{B}$ the beam on prompt neutron (6), and $N_{s S}$ the steady-steady state background (405). Expected number of new interaction event is represented by $N_{\text {exp }}$. Parameter $a$ and $b$ are the uncertainty of the pull parameters. Uncertainty for each case are $\sigma_{a}=0.28$ and $\sigma_{b}=0.25$. All of these parameters are obtained from the first result of the first COHERENT data [6].

Using Eq.21, we analyze the lower bound from each new mediator contributions in the parameter space of its mass and square of its coupling. We consider the same coupling strength to the neutrino and quarks constituent of the nucleus. For this activity, we develop CEvNS package from Ref.[17]. Fig. 4 shows the 90\% C.L. constraints of our results. In general, the coupling independents of its corresponding mass up to $10 \mathrm{MeV}$. Beyond this scale, mass dependency occurs. Particular to the vectorial case, the empty space is the region where degenerate between the vectorial interactions and the SM takes place. We notice that the vector and scalar cases are frequently studied recently and our results show consistency with Ref.[13].

\section{CONCLUSION}

We have shown the effect of the general interaction induced from a simplified model in the CEvNS process. Using the flux from reactor and accelerator neutrino experiment, event rates of the SM, as well as the new physics contribution, as the function of nuclear threshold energy are presented. It can be seen that the reactor neutrino results indicate a greater spectrum than from the accelerator, hence makes it more promising for searching the light new mediator's effect on CEvNS framework. Using the first COHERENT data, we have provided the $90 \%$ C.L. lower bound for the square of new couplings with their masses.

\section{References}

[1] D. Z. Freedman, Phys. Rev. D 9 (1974), 1389-1392.

[2] K. Scholberg, Phys. Rev. D 73 (2006), 033005.

[3] J. R. Wilson, Phys. Rev. Lett. 32 (1974), 849-852.

[4] A. J. Anderson, J. M. Conrad, E. Figueroa-Feliciano, et al., Phys. Rev. D 84 (2011), 013008.

[5] D. Akimov et al. (COHERENT), Science 357 (2017) no.6356, 1123-1126.

[6] D. Akimov et al. (COHERENT), [arXiv:1804.09459 [nucl-ex]].

[7] S. Kerman et al. (TEXONO), Phys. Rev. D 93 (2016) no.11, 113006.

[8] V. A. Bednyakov and D. V. Naumov, Phys. Rev. D 98 (2018) no.5, 053004.

[9] H. T. Wong, Nucl. Phys. A 844 (2010), 229C-233C.

[10] V. Sharma and H. T. Wong, J. Phys. Conf. Ser. 1468 (2020) no.1, 012149.

[11] P. Agnes et al. (DarkSide), Phys. Rev. Lett. 121 (2018) no.8, 081307.

[12] J. Hakenmüller, et al. (CONUS), Eur. Phys. J. C 79 (2019) no.8, 699.

[13] D. K. Papoulias, Phys. Rev. D 102 (2020) no.11, 113004.

[14] A. N. Khan and W. Rodejohann, Phys. Rev. D 100 (2019) no.11, 113003.

[15] M. Cadeddu, N. Cargioli, F. Dordei, et al., JHEP 01 (2021), 116.

[16] J. Billard, R. Carr, J. Dawson, et al. J. Phys. G 44 (2017) no.10, 105101.

[17] J. Billard, J. Johnston, and B. J. Kavanagh, JCAP 11 (2018), 016.

[18] M. Lindner, W. Rodejohann, and X. J. Xu, JHEP 03 (2017), 097.

[19] M. Tanabashi et al. (Particle Data Group), Phys. Rev. D 98 (2018) no.3, 030001.

[20] R. H. Helm, Phys. Rev. 104 (1956), 1466-1475.

[21] M. Cirelli, E.D. Nobile, and P. Panci, JCAP 04 (2013), 019.

[22] M. Hoferichter, J.R. de Elvira, B. Kubis, and U. Meißner, Phys. Rev. Lett. 115 (2015), 092301.

[23] P. Junnarkar and A. Walker-Loud, Phys. Rev. D 87 (2013), 114510.

[24] G. Belanger, F. Boudjema, A. Pukhov, and A. Semenov, Comput. Phys. Commun. 180 (2009), 747-767.

[25] J.R. Ellis, A. Ferstl, and K.A. Olive, Phys.Lett.B 481 (2000), 304-314. 